MATHEMATICS OF COMPUTATION

Volume 68, Number 228, Pages 1623-1630

S 0025-5718(99)01091-1

Article electronically published on May 17, 1999

\title{
VALUES OF THE LEGENDRE CHI AND HURWITZ ZETA FUNCTIONS AT RATIONAL ARGUMENTS
}

\author{
DJURDJE CVIJOVIĆ AND JACEK KLINOWSKI
}

Abstract. We show that the Hurwitz zeta function, $\zeta(\nu, a)$, and the Legendre chi function, $\chi_{\nu}(z)$, defined by

$$
\zeta(\nu, a)=\sum_{k=0}^{\infty} \frac{1}{(k+a)^{\nu}}, \quad 0<a \leq 1, \operatorname{Re} \nu>1
$$

and

$$
\chi_{\nu}(z)=\sum_{k=0}^{\infty} \frac{z^{2 k+1}}{(2 k+1)^{\nu}}, \quad|z| \leq 1, \operatorname{Re} \nu>1 \text { with } \nu=2,3,4, \ldots,
$$

respectively, form a discrete Fourier transform pair. Many formulae involving the values of these functions at rational arguments, most of them unknown, are obtained as a corollary to this result. Among them is the further simplification of the summation formulae from our earlier work on closed form summation of some trigonometric series for rational arguments. Also, these transform relations make it likely that other results can be easily recovered and unified in a more general context.

\section{INTRODUCTION}

Consider the series

$$
S_{\nu}(x)=\sum_{k=0}^{\infty} \frac{\sin ((2 k+1) \pi x)}{(2 k+1)^{\nu}} \quad \text { and } \quad C_{\nu}(x)=\sum_{k=0}^{\infty} \frac{\cos ((2 k+1) \pi x)}{(2 k+1)^{\nu}} \operatorname{Re} \nu>1
$$

Observe that the definitions of $S_{\nu}(x)$ and $C_{\nu}(x)$ ensure the convergence of each of the series involved: both series converge uniformly for all real values of $x$ when $\operatorname{Re} \nu>1 . C_{\nu}(x)$ and $S_{\nu}(x)$ are even and odd in $x$, respectively, while

$$
C_{\nu}(x)=-C_{\nu}(1-x) \text { and } S_{\nu}(x)=S_{\nu}(1-x) .
$$

Because of these properties, it is sufficient to calculate $C_{\nu}(x)$ and $S_{\nu}(x)$ over the range $0 \leq x \leq \frac{1}{2}$ only.

In practice, the series in (1) for $\nu=2,3,4, \ldots$ are of particular interest. It is well known that $S_{2 n+1}(x)$ and $C_{2 n}(x)$ are summable in terms of the Euler polynomials (see the equations in (10)). However, no simple summation formulae exist for $S_{2 n}(x)$ and $C_{2 n+1}(x)$, which are usually expressed in terms of certain integrals or even a combination of integrals.

Received by the editor February 16, 1998.

1991 Mathematics Subject Classification. Primary 65B10; Secondary 11M35.

Key words and phrases. Summation of series, Hurwitz's zeta function, Legendre's chi function.

(C)1999 American Mathematical Society 
Numerical evaluation of $S_{2 n}(x)$ and $C_{2 n+1}(x)$ is difficult, and the problem has recently been addressed in some detail (see [1]-[4]). For the particular case when $n=1$, Dempsey et al. [1] developed a procedure based on Plana's summation formula along with Romberg's method of integration, which significantly improves the convergence and accuracy in comparison with direct summation. Boersma and Dempsey [3] transformed $S_{2 n}(x)$ and $C_{2 n+1}(x)$ into a rapidly convergent series well suited for their computation. In particular, computation for $n=1$ was considered. Cvijović and Klinowski [4] showed that when $x$ is a rational, the series $C_{\nu}(x)$ and $S_{\nu}(x)$ in (1) can, in the general case, be summed in closed form in terms of the Hurwitz zeta function. In other words, numerical evaluation of these trigonometric series, when $x$ is a rational, is transformed into the evaluation of the series of a rational function, which is much easier numerically.

In this work we deduce that the Legendre chi function and the Hurwitz zeta function form a discrete Fourier transform pair. Among the formulae which are obtained as corollaries to this transform relation are the summation formulae found earlier for $C_{\nu}(x)$ and $S_{\nu}(x)$ at rational arguments, which are simplified further. Also, the relation makes it possible that other results can be easily recovered and unified in a more general context.

\section{The Legendre Chi FunCtion AND THE DISCRETE FOURIER TRANSFORM}

Recall the definition of the discrete Fourier transform (DFT) [5, Chapter 8]. Let $\left(a_{r}\right)(r=0,1, \ldots, t-1 ; t \geq 1)$ be a periodic sequence of real or complex numbers with period $t\left(a_{r+t}=a_{r}\right.$ for all $\left.r \in N_{0}\right)$. Then, the discrete Fourier transform pair of the sequences $\left(a_{r}\right)$ and $\left(a_{s}^{*}\right)$ is defined as

$$
a_{s}^{*}=\frac{1}{t} \sum_{r=0}^{t-1} a_{r} w^{-r s}, \quad s=0,1, \ldots, t-1,
$$

where $w=\exp (i 2 \pi / t)$

$$
a_{r}=\sum_{s=0}^{t-1} a_{s}^{*} w^{r s}, \quad r=0,1, \ldots, t-1 .
$$

The first relation is known as the direct discrete Fourier transform, and the second as the inverse discrete Fourier transform. We note that it is usually asserted that $0 \leq r \leq t-1$ and $0 \leq s \leq t-1$, but $r$ and $s$ can be arbitrary integers (or residues modulo $t$ ). We shall also need the following equation known as the orthogonality relationship:

$$
\sum_{r=1}^{t} w^{r s} w^{-r s}=\left\{\begin{array}{ll}
t & \text { if } r=s, \\
0 & \text { otherwise }
\end{array}\right\} \quad s=0,1, \ldots, t-1 .
$$

The Riemann and the Hurwitz zeta functions, $\zeta(\nu)$ and $\zeta(\nu, a)$, respectively, are defined by the series $[6$, pp. 19, 22]

$$
\zeta(\nu)=\sum_{k=1}^{\infty} \frac{1}{k^{\nu}} \quad \text { and } \quad \zeta(\nu, a)=\sum_{k=0}^{\infty} \frac{1}{(k+a)^{\nu}}, \quad 0<a \leq 1, \operatorname{Re} \nu>1 .
$$

It is evident that $\zeta(\nu)=\zeta(\nu, 1)$. 
The series

$$
\chi_{\nu}(z)=\sum_{k=0}^{\infty} \frac{z^{2 k+1}}{(2 k+1)^{\nu}}, \quad|z| \leq 1, \operatorname{Re} \nu>1,
$$

in the particular case when $\nu=n=2,3,4, \ldots$, is usually referred to as the Legendre chi function of order $n$ [7, p. 283, Eq. A1.29]. The theory of this and related functions is thoroughly covered in Lewin's standard text [7].

Having introduced all the definitions we need, we state the following theorem.

Theorem (DFT pair of $\zeta(\nu, a)$ and $\left.\chi_{\nu}(z)\right)$. Assume that $t$ is a positive integer and set $\omega=\exp (i \pi / t)$. Let $\zeta(\nu, a)$ and $\chi_{\nu}(z)$ be the Hurwitz zeta function and the Legendre chi function defined as in (5) and (6). Then

$$
\zeta(\nu,(2 s-1) / 2 t)=\frac{1}{t} \sum_{r=1}^{t}(2 t)^{\nu} \chi_{\nu}\left(\omega^{r}\right) \omega^{-r(2 s-1)}, \quad s=1,2, \ldots, t,
$$

and

$$
\chi_{\nu}\left(\omega^{r}\right)=\frac{1}{(2 t)^{\nu}} \sum_{s=1}^{t} \zeta(\nu,(2 s-1) / 2 t) \omega^{r(2 s-1)}, \quad r=1, \ldots, t .
$$

The formula in $(7 \mathrm{~b})$ shall be derived first; i.e., it will be shown that the sequence $(2 t)^{\nu} \chi_{\nu}\left(\omega^{r}\right)$ is the inverse Fourier transform of $\zeta(\nu,(2 s-1) / 2 t)$. We begin by noting that absolute convergence of the series

$$
\chi_{\nu}\left(\omega^{r}\right)=\sum_{k=0}^{\infty} \frac{\exp [i(2 k+1) \pi r / t]}{(2 k+1)^{\nu}}=\omega^{-r} \sum_{k=0}^{\infty} \frac{\exp [i 2(k+1) \pi r / t]}{(2 k+1)^{\nu}}, \quad 1 \leq r \leq t,
$$

is assured when $\operatorname{Re} \nu>1$. It is clear that the sequence of the numbers $\chi_{\nu}\left(\omega^{r}\right)$, and thus the sequence $(2 t)^{\nu} \chi_{\nu}\left(\omega^{r}\right)$, are periodic with respect to $r$ with period $t$.

Next, recall that for any $a \in Z, b \in N$ there exist unique $c, d \in Z$ such that $a=b c+d$ and $0 \leq d<b$ (division law in $Z$ ). Here, this means that any $(k, t)$ $\left(k \in N_{0}, t \in N\right)$ uniquely determine the integers $m$ and $s$ such that $k=t m+s$, where $m=0,1,2, \ldots$ and $s=0,1, \ldots, t-1$. Hence, it follows by absolute convergence that

$$
\begin{aligned}
\chi_{\nu}\left(\omega^{r}\right) \omega^{r} & =\sum_{m=0}^{\infty} \sum_{s=0}^{t-1} \frac{\exp [i 2(t m+s+1) \pi r / t]}{[2 t m+2 s+1]^{\nu}} \\
& =\sum_{m=0}^{\infty} \sum_{s=1}^{t} \frac{\exp [i 2(t m+s) \pi r / t]}{[2 t m+2 s-1]^{\nu}} \\
& =\frac{1}{(2 t)^{\nu}} \sum_{s=1}^{t} \sum_{m=0}^{\infty} \frac{\exp (i 2 m \pi r) \exp (i 2 s \pi r / t)}{[m+(2 s-1) / 2 t]^{\nu}},
\end{aligned}
$$

which can be further simplified to

$$
\begin{aligned}
\chi_{\nu}\left(\omega^{r}\right) & =\frac{\omega^{-r}}{(2 t)^{\nu}} \sum_{s=1}^{t} \sum_{m=0}^{\infty} \frac{\exp (i 2 s \pi r / t)}{[m+(2 s-1) / 2 t]^{\nu}} \\
& =\frac{1}{(2 t)^{\nu}} \sum_{s=1}^{t} \exp [i(2 s-1) \pi r / t] \sum_{m=0}^{\infty} \frac{1}{[m+(2 s-1) / 2 t]^{\nu}}
\end{aligned}
$$


since $\exp (i 2 m \pi r)=1$ ( $m$ and $r$ are integers). In view of the definition of the Hurwitz zeta function in (5), the last double sum results in the required formula in (7b).

We will now show that the relations in (7a) and (7b) form a discrete Fourier pair. Indeed, the substitution of (7b) into (7a) yields

$$
\begin{aligned}
\zeta(\nu,(2 s-1) / 2 t)=\frac{1}{t} \sum_{r=1}^{t}(2 t)^{\nu} \chi_{\nu}\left(\omega^{r}\right) \omega^{-r(2 s-1)} \\
=\frac{1}{t} \sum_{r=1}^{t}(2 t)^{\nu}\left((2 t)^{-\nu} \sum_{s=1}^{t} \zeta(\nu,(2 s-1) / 2 t) \omega^{r(2 s-1)}\right) \omega^{-r(2 s-1)} \\
=\frac{1}{t} \sum_{s=1}^{t} \zeta(\nu,(2 s-1) / 2 t) \sum_{r=1}^{t} \omega^{-r(2 s-1)} \omega^{r(2 s-1)} \\
=\zeta(\nu,(2 s-1) / 2 t), \quad 1 \leq s \leq t,
\end{aligned}
$$

since by (4) we have

$$
\sum_{r=1}^{t} \omega^{r(2 s-1)} \omega^{-r(2 s-1)}=\sum_{r=1}^{t} \omega^{2 r s} \omega^{-2 r s}=\left\{\begin{array}{ll}
t, & \text { if } r=s, \\
0, & \text { otherwise },
\end{array}\right\}
$$

considering that $w=\omega^{2}$. In this way, the proposed discrete Fourier transform relations in (7a) and (7b) are established for $\operatorname{Re} \nu>1$ and our theorem is proved.

\section{VARIOUS FORMUlAE}

Recall that the Euler polynomial of degree $n$, denoted by $E_{n}(x)$, is defined as

$$
E_{n}(x)=\sum_{k=0}^{n}\left(\begin{array}{l}
n \\
k
\end{array}\right) E_{k} 2^{-k}\left(x-\frac{1}{2}\right)^{n-k} \quad n=0,1,2, \ldots
$$

[6, p. 39], where the rational numbers $E_{k}$ are the Euler numbers given by the coefficients in the power series

$$
\frac{1}{\cos h t}=\sum_{k=0}^{\infty} E_{k} \frac{t^{k}}{k !} \quad|t|<\pi / 2 .
$$

Let $S_{\nu}(x)$ and $C_{\nu}(x)$ be the series defined in (1), then $E_{n}(x)$ is represented by the following Fourier series [8, p. 805, Eq. 23.1.17 and 23.1.18]:

$$
C_{2 n}(x)=\frac{(-1)^{n}}{4(2 n-1) !} \pi^{2 n} E_{2 n-1}(x),
$$

where $0 \leq x \leq 1$ for $n=1,2,3, \ldots$, and

$$
S_{2 n+1}(x)=\frac{(-1)^{n}}{4(2 n) !} \pi^{2 n+1} E_{2 n}(x),
$$

where $0 \leq x \leq 1$ for $n=1,2,3, \ldots, 0<x<1$ for $n=0$.

We now give several immediate consequences of our theorem for the relation between the Hurwitz zeta function $\zeta(\nu, a)$ and the Legendre chi function $\chi_{\nu}(z)$. In what follows, $n$ and $q$ are positive integers, $p$ is a integer, and $\nu$ is a complex number with $\operatorname{Re} \nu>1$. 
First, in view of the definition in (6), we see that the infinite series $S_{\nu}(x)$ and $C_{\nu}(x)$ in (1) can be considered as the imaginary and real parts

$$
S_{\nu}(x)=\operatorname{Im}\left[\chi_{\nu}(\exp (i x))\right], \quad C_{\nu}(x)=\operatorname{Re}\left[\chi_{\nu}(\exp (i x))\right],
$$

of $\chi_{\nu}(\exp (i x))$. Thus, the following summation formulae,

$$
S_{\nu}(p / q)=\frac{1}{(2 q)^{\nu}} \sum_{s=1}^{q} \zeta(\nu,(2 s-1) / 2 q) \sin ((2 s-1) \pi p / q)
$$

and

$$
C_{\nu}(p / q)=\frac{1}{(2 q)^{\nu}} \sum_{s=1}^{q} \zeta(\nu,(2 s-1) / 2 q) \cos ((2 s-1) \pi p / q),
$$

which enable a closed-form evaluation of the series $S_{\nu}(x)$ and $C_{\nu}(x)$ in (1) for rational arguments $(x=p / q$ with $0 \leq p \leq q)$, are readily available from $(7 \mathrm{~b})$.

Second, we have that the values of the Euler polynomial $E_{n}(x)$ of degree $n \geq 1$ at rational arguments $(x=p / q$ with $0 \leq p \leq q)$ are given by

$$
E_{2 n-1}(p / q)=(-1)^{n} \frac{4(2 n-1) !}{(2 \pi q)^{2 n}} \sum_{s=1}^{q} \zeta(2 n,(2 s-1) / 2 q) \cos ((2 s-1) \pi p / q)
$$

and

$$
E_{2 n}(p / q)=(-1)^{n} \frac{4(2 n) !}{(2 \pi q)^{2 n+1}} \sum_{s=1}^{q} \zeta(2 n+1,(2 s-1) / 2 q) \sin ((2 s-1) \pi p / q) .
$$

These formulae are obtained by combining (9a), (9b) and (11a), (11b).

Third, in view of (10), the formula in (7a) can be rewritten as

$$
\begin{aligned}
\zeta(\nu,(2 p-1) / 2 q)= & 2(2 q)^{\nu-1} \sum_{r=1}^{q}\left(\Phi_{1}(r)+i \Phi_{2}(r)\right)=2(2 q)^{\nu-1} \sum_{r=1}^{q} \Phi_{1}(r) \\
= & 2(2 q)^{\nu-1} \sum_{r=1}^{q} C_{\nu}(r / q) \cos ((2 p-1) \pi r / q) \\
& \quad+2(2 q)^{\nu-1} \sum_{r=1}^{q} S_{\nu}(r / q) \sin ((2 p-1) \pi r / q), \quad 1 \leq p \leq q,
\end{aligned}
$$

given that

$$
\sum_{r=1}^{q} \Phi_{2}(r)=\sum_{r=1}^{q-1} \Phi_{2}(r)=0
$$

where

$$
\Phi_{2}(r)=S_{\nu}(r / q) \cos ((2 p-1) \pi r / q)-C_{\nu}(r / q) \sin ((2 p-1) \pi r / q), \quad 1 \leq r \leq q,
$$

since, on making use of (2), we conclude that $\Phi_{2}(q)=0$ and $\Phi_{2}(q-r)=\Phi_{2}(q)$ $(1 \leq r \leq q-1)$. Moreover, putting $2 q-(2 p-1)$ in (13a) instead of $2 p-1$ results 
in

$$
\begin{aligned}
\zeta(\nu, 1- & (2 p-1) / 2 q) \\
= & 2(2 q)^{\nu-1} \sum_{r=1}^{q} C_{\nu}(r / q) \cos ((2 p-1) \pi r / q) \\
& -2(2 q)^{\nu-1} \sum_{r=1}^{q} S_{\nu}(r / q) \sin ((2 p-1) \pi r / q), \quad 1 \leq p \leq q .
\end{aligned}
$$

Finally, for $1 \leq p \leq q$, the fourth set

$$
\zeta(\nu,(2 p-1) / 2 q)+\zeta(\nu, 1-(2 p-1) / 2 q)
$$

and the fifth set

$$
\begin{aligned}
& =4(2 q)^{\nu-1} \sum_{r=1}^{q} C_{\nu}(s / q) \cos ((2 p-1) \pi r / q), \\
\zeta(\nu,(2 p-1) / 2 q)-\zeta(\nu, 1-(2 p-1) / 2 q) & \\
= & 4(2 q)^{\nu-1} \sum_{r=1}^{q} S_{\nu}(r / q) \sin ((2 p-1) \pi r / q),
\end{aligned}
$$

$$
\begin{aligned}
& \zeta(2 n,(2 p-1) / 2 q)+\zeta(2 n, 1-(2 p-1) / 2 q) \\
& =(-1)^{n} \pi^{2 n} \frac{(2 q)^{2 n}}{(2 n-1) !} \sum_{r=1}^{q} E_{2 n-1}(r / q) \cos ((2 p-1) \pi r / q), \\
& \zeta(2 n+1,(2 p-1) / 2 q)-\zeta(2 n+1,1-(2 p-1) / 2 q) \\
& =(-1)^{n} \pi^{2 n+1} \frac{(2 q)^{2 n}}{(2 n) !} \sum_{r=1}^{q} E_{2 n}(r / q) \sin ((2 p-1) \pi r / q)
\end{aligned}
$$

of the formula follow at once from (13a), (13b) and (9a), (9b), respectively, in conjunction with (13a) and (13b).

\section{Discussion and special values}

It does not seem to have been noticed earlier that the discrete Fourier transform relation exists, which we have established in the above theorem, between the Legendre chi function and the Hurwitz zeta function. Also, we have failed to find in the literature our formulae given in (13a), (13b) through (15a), (15b), here easily obtained as corollaries. Recall, however, the following well-known relations $[6, \mathrm{p}$. 260, Eq. 6.4.7 in conjunction with Eq. 6.4.10]

$$
\begin{aligned}
& \zeta(2 n+1, x)-\zeta(2 n+1,1-x)=\frac{\pi}{(2 n) !} \cot (\pi x)^{(2 n)}, \\
& \zeta(2 n, x)+\zeta(2 n, 1-x)=-\frac{\pi}{(2 n-1) !} \cot (\pi x)^{(2 n-1)},
\end{aligned}
$$

which involve the derivatives of $\cot (\pi x)$ and are valid when $n \geq 1,0<x<1$.

Observe that the problem of closed-form summation of the series $S_{\nu}(x)$ and $C_{\nu}(x)$ for rational $x$ was settled in our earlier work [4, Equations 8 and 9], where various related special results were also established. Note, however, that a comparison of Equations 8 and 9 and the summation formulae in (11a), (11b) above shows that (i) the formulae given in Equation 8 are more complicated than those in (11a), (11b), 
and (ii) the formulae in Equation 9 are derived on the assumption that $p$ is odd and $q$ even, while there are no such restrictions for (11a), (11b). Finally, note that the formulae for the Euler polynomials at rational arguments in (12a), (12b) were deduced earlier [9, Theorem B].

To conclude, we give several illustrative examples. First, in view of [9, Eq. 12a]

$$
\sum_{s=1}^{q} \zeta(\nu, s / q)=q^{\nu} \zeta(\nu)
$$

the following relations hold:

$$
\begin{gathered}
\zeta\left(\nu, \frac{1}{4}\right)+\zeta\left(\nu, \frac{3}{4}\right)=2^{\nu}\left(2^{\nu}-1\right) \zeta(\nu), \\
\zeta\left(\nu, \frac{1}{6}\right)+\zeta\left(\nu, \frac{5}{6}\right)=\left(2^{\nu}-1\right)\left(3^{\nu}-1\right) \zeta(\nu) .
\end{gathered}
$$

Then, for instance, the formula in (11b) in conjunction with (16b) readily gives

$$
C_{\nu}(1 / 3)=-C_{\nu}(2 / 3)=(1 / 2)\left(2^{-\nu}-1\right)\left(3^{1-\nu}-1\right) .
$$

Further, it is an easy exercise to verify the following well-known result $[8$, p. $803-$ 806]:

$$
E_{2 n-1}(1 / 3)=-E_{2 n-1}(2 / 3)=(1 / 2 n)\left(2^{2 n}-1\right)\left(3^{1-2 n}-1\right) B_{2 n},
$$

where $B_{2 n}$ are the even-indexed Bernoulli numbers $B_{2 n}$ given by the Euler relation

$$
B_{2 n}=(-1)^{n-1} \frac{2(2 n) !}{(2 \pi)^{2 n}} \zeta(2 n) .
$$

Indeed, starting from (12a) on making use of (16b) and the Euler relation we have

$$
\begin{aligned}
E_{2 n-1}(1 / 3) & =(-1)^{n} \frac{4(2 n-1) !}{(2 \pi)^{2 n}} \frac{1}{3^{2 n}}[(1 / 2)(\zeta(2 n, 1 / 6)+\zeta(2 n, 5 / 6))-\zeta(2 n, 1 / 2)] \\
& =(-1)^{n} \frac{4(2 n-1) !}{(2 \pi)^{2 n}} \frac{1}{3^{2 n}}(3 / 2)\left(2^{2 n}-1\right)\left(3^{2 n-1}-1\right) \zeta(2 n) \\
& =(1 / 2)\left(2^{2 n}-1\right)\left(3^{1-2 n}-1\right)(-1)^{n} \frac{4(2 n-1) !}{(2 \pi)^{2 n}} \zeta(2 n) \\
& =(1 / 2 n)\left(2^{2 n}-1\right)\left(3^{1-2 n}-1\right) B_{2 n} .
\end{aligned}
$$

Finally, if $n$ is a positive integer we have

$$
\begin{aligned}
2 \zeta\left(2 n+1, \frac{1}{4}\right) & =2^{2 n+1}\left(2^{2 n+1}-1\right) \zeta(2 n+1)+\sqrt{2} E_{2 n} \frac{1}{4} 4^{2 n} Q_{n}, \\
2 \zeta\left(2 n+1, \frac{3}{4}\right) & =2^{2 n+1}\left(2^{2 n+1}-1\right) \zeta(2 n+1)+\sqrt{2} E_{2 n} \frac{1}{4} 4^{2 n} Q_{n},
\end{aligned}
$$

where $Q_{n}$ is given by

$$
Q_{n}=(-1)^{n} \frac{(2 \pi)^{2 n+1}}{2(2 n) !},
$$

which follows at once on using (16a) and our formula in (15b). 


\section{REFERENCES}

1. K. M. Dempsey, D. Liu and J. P. Dempsey, Plana's summation formula for $\sum m^{-2} \sin (m \alpha)$, $m^{-3} \cos (m \alpha), m^{-2} A^{m}, m^{-3} A^{m}$, Math. Comp. 55 (1990), 693-703. MR 91b:65003

2. W. Gautschi, On certain slowly convergent series occurring in plate contact problems, Math. Comp. 57 (1991), 325-338. MR 91j:40002

3. J. Boersma and J. P. Dempsey, On the numerical evaluation of Legendre's chi-function, Math. Comp. 59 (1992), 157-163. MR 92k:65008

4. D. Cvijović and J. Klinowski, Closed-form summation of some trigonometric series, Math. Comp. 64 (1995), 205-210. MR 95f:65017

5. H. J. Weaver, Theory of discrete and continuous Fourier analysis, John Wiley, New York, 1989. MR 90c: 42002

6. W. Magnus, F. Obergettinger and R. P. Soni, Formulas and theorems for the special functions of mathematical physics, Springer-Verlag, Berlin, 1966. MR 38:1291

7. L. Lewin, Polylogarithms and associated functions, North-Holland, Amsterdam, 1981. MR 83b:33019

8. M. Abramowitz and I. Stegun (eds.), Handbook of mathematical functions with formulas, graphs and mathematical tables, U. S. Government Printing Office, 1966. MR 34:8607

9. D. Cvijović and J. Klinowski, New formulae for the Bernoulli and Euler polynomials at rational arguments, Proc. Amer. Math. Soc. 123 (1995), 1527-1535. MR 95g:11085

Department of Chemistry, University of Cambridge, Lensfield Road, Cambridge CB2 1EW, United Kingdom

E-mail address: dc133@cam.ac.uk

E-mail address: jk18@cam.ac.uk 